\title{
On the Information Freshness and Tail Latency Trade-off in Mobile Networks
}

\author{
Abubakr O. Al-Abbasi ${ }^{1}$, Ali Elghariani ${ }^{1}$, Anis Elgabli ${ }^{2}$, and Vaneet Aggarwal $^{1}$ \\ ${ }^{1}$ Purdue University, ${ }^{2}$ University of Oulu
}

\begin{abstract}
With the advent of emerging mission-critical applications, sampling information updates and scheduling mobile traffic in a timely manner are very challenging. In addition, maintaining fresh information and low latency communication is important to these applications. To that end, in this paper, we first derive closed form expressions for an upper bound on the latency tail probability (LTP) and the average age of information (AoI) in M/G/1 systems, where shifted exponential service time is considered. Different from the majority of existing work in this domain, our analysis is derived under different update size assumption with different priority levels. Next, we have developed novel policies for sampling and scheduling the information updates over the choice of one of the parallel links, e.g., WiFi and LTE links. Then, a joint minimization of AoI and LTP is formulated and efficient algorithms are provided. Our evaluation results show that our proposed approaches outperform the state-of-the-art algorithms and some competitive baselines.

Index Terms-Age of information, Real time application, Tail probability, Latency, Sampling, Scheduling.
\end{abstract}

\section{INTRODUCTION}

The continuous increase in the number of smart phones has led to a rapid growth in mobile services and applications. With the advent of timely-mission-critical emerging applications, sampling information updates and scheduling wireless traffic and information updates in a timely manner are very challenging. This is because conventional techniques of sampling and/or scheduling have been mainly designed to achieve high throughput and small delay [1]. Nonetheless, these performance measures fail to capture the time sensitivity of the information from the application perspective which is important for real-time and IoT (Internet-of-things) applications.

The freshness of delivered information is measured by a recently proposed metric, called Age of Information (AoI) [2], [3]. AoI is typically defined as the time elapsed since the last delivered information was generated (at the source). In contrast to packet-centric measures like throughput or latency, AoI is a destination-centric metric which makes it more appropriate to characterize the freshness and timeliness of information updates. We note that AoI is fundamentally different from traditional performance measures such as throughput and latency. To reduce the latency, information updates should not be sent frequently to reduce the system overload and avoid system congestion (i.e., resulting in a low latency). However, as captured in Figure 1, the information at the destination could be stale due to a lack of fresh updates. Intuitively, the higher the rate updates, the fresh the information is (i.e., age will be small since more fresh updates are executed and delivered).

Recently, AoI received great attention to study the freshness of the rapid deployment applications [3]. Such applications include vehicular networks [2], feedback for wireless channels [4], cloud gaming and mobile caching [5]. In addition, imposing deadlines on information updates, sampling cost, computation cost of updates have been extensively investigated in [6]-[8]. In [9], similar analysis for characterizing AoI for a single link was performed under different queueing disciplines including $\mathrm{M} / \mathrm{M} / 1, \mathrm{M} / \mathrm{D} / 1$, and $\mathrm{D} / \mathrm{M} / 1$. Further, age was investigated for different arrivals and service time distributions in several studies, see for example [10] and references therein. The tail and mean AoI tradeoff is studied in [11]. The response time and peak-AoI tradeoff is considered in [12], [13]. However, peak-AoI and/or a single source and single server are assumed. Various modeling and scheduling techniques have been proposed for quantifying latency in different queuing settings [14], [15]. In [16], mean service latency is studied for replication based systems for homogeneous servers with independent exponential service times. In [17], [18], Forkjoin policy is used to provide upper bounds for mean service latency by forking each file request to all storage servers.

Despite the great attention to study the freshness of the delivered information for mobile services and rapid deployment applications [3], [19], there is no work, to the best of our knowledge, that takes into its account the different time-sensitivity levels among different applications and jointly optimizing the AoI and the latency tail probability (LTP), which is the focus of this paper. The LTP of an update of a certain application is defined as the probability that the latency is greater than a certain threshold. We note that the tail probability of latency has been shown to affect the user experience much more than the mean [20], [21], and that motivates us to consider the tail metric.

In this paper, we consider a scenario in which multiple applications (apps), running on a mobile device, are competing the available resources to send their most recent information to a remote node (e.g., BS) ${ }^{1}$. One way to minimize the AoI of the applications is by properly sampling the information of the apps and efficiently exploiting the multiple orthogonal links of the mobile device. Wireless devices are equipped with multiple links such as LTE and WiFi. The scheduled application can

\footnotetext{
${ }^{1}$ Our framework is applicable to a wide range of other information-update systems including cyber physical systems and distributed/cloud computing.
} 
use one of these links to send the information. However, scheduling the updates of the apps over the parallel links is a challenging task since an optimal policy should consider the queue status, weight/importance of each update, and the updates that are not fully served when taking the dispatching policy. To overcome these problems, we propose a scheduling policy, so-called, Probabilistic Sampling and Scheduling (PSS) which works as follows. First, the information updates of the source are sampled with certain probability. Second, the sampled informations of the different apps are probabilistically scheduled over the two mobile links. In contrast to many queue-based scheduling techniques such as join the shortest queue (JSQ), power-of-d Pof(d), and least load (LL) [22][24], where the instantaneous queue level is kept tracked, our scheduling policy is independent of the instantaneous queue level and employs only the average queue length in its decision. Further, PSS can differentiate and provide different priority levels among the update packets so that updates with critical timing can be prioritized over the others.

We aim to fundamentally understand the tradeoff between LTP and freshness of information. Towards this goal, we first quantify the LTP and AoI in closed forms and then formulate an optimization problem to jointly minimize a weighted sum of both AoI and LTP. The key parameters are the rate at which the information updates are sampled and the scheduling probabilities over both WiFi and LTE links. The optimization problem is approximated as a convex problem and solved efficiently using successive convex approximation. Evaluation results demonstrate a significant improvement of AoI metric as compared to the state of the art algorithms, such as JSQ, LL(d), and Pof(d), and some competitive baselines.

\section{SySTEM MODEL}

We consider $N$ different applications running on a mobile device, each of which tries to send its most fresh information updates to the base station (BS) or a remote monitor, as depicted in Figure 2. We assume that every application sends its recent data asynchronously to the receiver to update its stored information. This information is updated frequently and the BS has to maintain the last updated information to perform certain decisions whenever required.

Let $i$ denote the $i$-th application, i.e, $i \in \mathbb{I}=\{1, \cdots, N\}$. Further, we consider that an application $i$ generates updates at a rate of $\widetilde{\lambda}_{i}$, the updates are sent using a wireless link $v \in \mathbb{V}=\{\mathrm{LTE}, \mathrm{WiFi}\}$. The rates of update generation are assumed to follow a Poisson process and are independent across different applications. Thus, the inter-update time (time between two consecutive updates, $X_{2}$ in Fig. 3), for every app $i$, is exponentially distributed with rate $\widetilde{\lambda}_{i}$. The update can be assigned to any link $v$ to transmit. Further, the service for data is assumed to be non-preemptive so tasks cannot be interrupted if they are already in service. In order to send the data of an app $i$, we first need to choose one link $v$ to serve the request and then send it to the BS.

The selection process of link $v$ is a challenging task as it needs to take into consideration many factors including the queue of each link, importance of every update as well as the running updates that are not fully executed yet. Besides those factors, the policy should also efficiently schedule the apps such that the age of information updates is minimized. In Section III-B, we will explain our proposed scheduling policy and will show how this policy is optimized to jointly reduce the LTP as well as the AoI of the updates for app $i, i \in N$. However, unlike [25], we assume that the update sizes of the apps are different, not identical ( $D_{i}$ for app $i$ ).

The service time of an app $i$ at link $v$ is assumed to follow a shifted exponential distribution with two parameters $\left(\beta_{v}, \alpha_{v}\right)$ [26]. Hence, the distribution of the service time for an update of app $i$ served through link $v$ is given by

$$
f_{v, i}(s)= \begin{cases}\alpha_{v, i} e^{-\alpha_{v, i}\left(s-\beta_{v, i}\right)} & s \geq \beta_{v, i} \\ 0 & s<\beta_{v, i}\end{cases}
$$

where $\alpha_{v, i}=\alpha_{v} / D_{i}$ and $\beta_{v, i}=\beta_{v} D_{i}$. Note that the transmission time of an update for app $i$ is $D_{i}\left(\beta_{v}+1 / \alpha_{v}\right)$, $\beta_{v}$ represents the shift and $\alpha_{v}$ represents the rate of the exponential random part. The value of $\alpha_{v, i}$ decreases in proportion to the update size while $\beta_{v, i}$ increases, in the exponential service time distribution. While the service time of each app update depends on the wireless channel, , we assume that the effects of the transmission medium are captured in the two components $\left(\alpha_{v}\right.$ and $\left.\beta_{v}\right)$ of the service model. The shift part represents the minimum of transmission time of source $i$ update to be successfully received at the monitor, which accounts also for the bandwidth and reliability of the transmission medium. Further, the exponential part captures the randomness in the transmission channel and the background running processes that make the transmission time non-deterministic. Unlike exponential service model which is widely considered in the literature, our shifted exponential model gives more flexibility to better model the service model in reality. To further clarify, one can draw the exponential distribution from the shifted exponential one by setting $\beta_{v, i}=0$. Similarly, deterministic service time can follow as a special case by taking the exponential rate very high. Let $M_{v, i}\left(t_{v}\right)=\mathbb{E}\left[e^{t_{v} S_{v, i}}\right]$ be the moment generating function (MGF) of the service time of an update at app $i$ at link $v$, $S_{v, i}$. Then, $M_{v, i}\left(t_{v}\right)$ is given as

$$
M_{v, i}\left(t_{v}\right)=\frac{\alpha_{v, i}}{\alpha_{v, i}-t_{v}} e^{\beta_{v, i} t_{v}}
$$

\section{Characterization OF Metrics}

\section{A. Probabilistic Sampling}

In this section, we describe our sampling policy for information updates. Every source generates new information with a rate of $\widetilde{\lambda}_{i}$. However, sending all the updates may lead to increased load at the transmission links, resulting in congested links. Therefore, we propose to under-sample the rate at which the information is queued and allow only $q_{i} \widetilde{\lambda}_{i}$, $0 \leq q_{i} \leq 1, \forall i$, to enter the queue of the link $v$, where $q_{i}$ is an optimization parameter. From Fig. 1, we see that choosing 


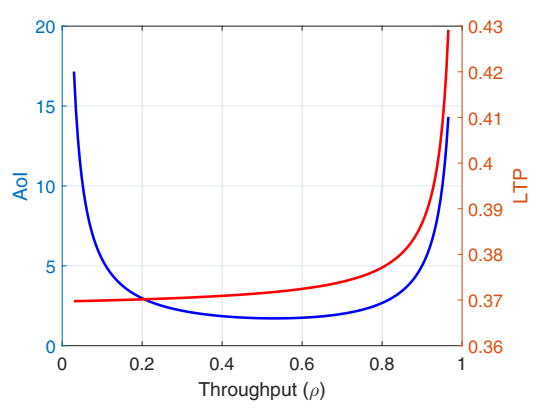

Fig. 1. AoI and LTP as functions of throughput, normalized by the service rate for $\delta_{i}=60$.

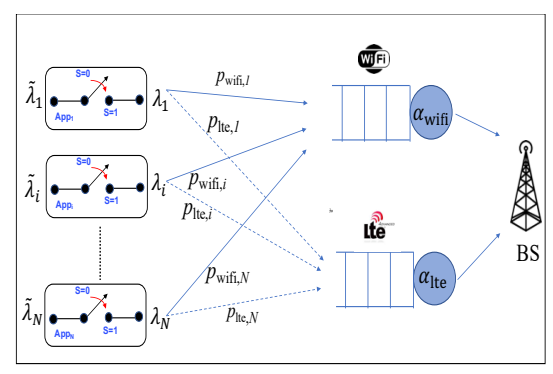

Fig. 2. A schematic illustrating one mobile device, with $N$ applications, equipped with WiFi and LTE links.

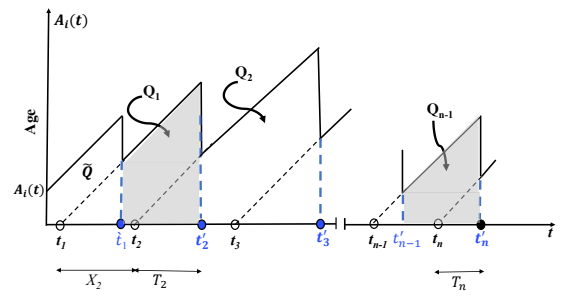

Fig. 3. An example illustrating the timeline of status updates of $A_{i}(t)$ at a remote monitor. Updates from source $i$ arrive at times marked with an empty circle and are received at the BS at times marked with a filled circle. the update rate affects both age and LTP. Hence, we propose a probabilistic sampling policy as follows. Each app $i$ is sampled with probability $q_{i}$. Under probabilistic sampling and since sampling Poisson process is also Poisson, the effective arrival rate of updates of an app $i$ forms a Poisson process with rate:

$$
\lambda_{i}=q_{i} \widetilde{\lambda}_{i}, \forall i
$$

\section{B. Uplink Probabilistic Scheduling}

We now describe a feasible scheduling policy for the app updates. Upon arrival of updates at the queue, LTE or WiFi interface has to be chosen to send the data. To provide prioritized service levels, we propose a prioritized probabilistic sampling and scheduling (i.e., PSS) policy as follows. Each interface $v$ has its own queue and the updates in each queue are served under First Come First Serve ${ }^{2}$. An update belong to app $i$ is assigned to the queue of interface $v \in \mathbb{V}=\{L T E, W i F i\}$, with probability $p_{v, i} \geq 0$ and for any update of app $i$, the following condition has to be satisfied for feasibility of scheduling process:

$$
\sum_{v \in\{L T E, W i F i\}} p_{v, i}=1
$$

Under PSS scheduling, the arrival of update requests at interface $v$ forms a Poisson process with rate:

$$
\Lambda_{v}=\sum_{i=1}^{N} p_{v, i} \lambda_{i}
$$

which is the superposition of $N$ Poisson processes each with rate $p_{i, v} \lambda_{i}$.

\section{Latency Tail Probability (LTP) Characterization}

Let $\mathbf{L}_{\mathbf{i}}$ be the random sojourn time that app $i$ needs it in order to deliver its data to the remote destination (e.g., BS). The LTP of an update for app $i$ is defined as the probability that $\mathbf{L}_{\mathbf{i}}$ is greater than or equal to $\delta_{i}$, for a given $\delta_{i}$. The service time of an update for app $i$ if served through link $v, S_{v, i}$, is

\footnotetext{
${ }^{2}$ While FCFS is considered, our approach can be extended to accommodate different queueing disciplines.
}

given by $\left(\beta_{v, i}+1 / \alpha_{v, i}\right)$. Hence, the service time at link $v$ is then expressed as

$$
S_{v}=\left\{S_{v, i} \quad \text { with probability } \frac{\lambda_{i} p_{v, i}}{\Lambda_{v}}, \forall \mathbf{i},\right.
$$

this is because the service time is $S_{v, i}$ which happens with probability $\frac{\lambda_{i} p_{v, i}}{\Lambda_{v}}$.

Lemma 1. The moment generating function for the service time of $S_{v}, M_{v}(t)=\mathbb{E}\left[e^{t S_{v}}\right]$, is given as

$$
M_{v}(t)=\sum_{i=1}^{N} \frac{p_{v, i} \lambda_{i}}{\Lambda_{v}}\left(\frac{\alpha_{v, i} e^{\beta_{v, i} t}}{\alpha_{v, i}-t}\right)
$$

for any $t>0$, and $t<\alpha_{v, i}$.

Proof. From (6), the service time of an update for app $i$, if served via link $v$, is $S_{v, i}$. Then, we can write

$$
M_{v}(t)=\sum_{i=1}^{N} \frac{p_{v, i} \lambda_{i}}{\Lambda_{v}} \mathbb{E}\left[e^{t S_{v, i}}\right] \stackrel{(a)}{=} \sum_{i=1}^{N} \frac{p_{v, i} \lambda_{i}}{\Lambda_{v}}\left(\frac{\alpha_{v, i} e^{\beta_{v, i} t}}{\alpha_{v, i}-t}\right)
$$

where (a) follows from (2).

Since $\mathbb{E}\left[S_{v}\right]=M_{v}^{\prime}(0)$, using Lemma 1 , the expected service time at link $v$ is given by

$$
\mathbb{E}\left[S_{v}\right]=\sum_{i=1}^{N} \frac{p_{v, i} \lambda_{i}}{\Lambda_{v}}\left(\beta_{v, i}+\frac{1}{\alpha_{v, i}}\right)
$$

Similarly, one can show that the utilization of link $v$ for app updates is given as

$$
\rho_{v}=\sum_{i=1}^{N} p_{v, i} \lambda_{i}\left(\beta_{v, i}+\frac{1}{\alpha_{v, i}}\right)
$$

Theorem 2. The LTP for an update of app $i, \operatorname{Pr}\left(\mathbf{L}_{\mathbf{i}} \geq \delta_{i}\right)$, using PSS scheduling is bounded by

$$
\operatorname{Pr}\left(\mathbf{L}_{\mathbf{i}} \geq \delta_{i}\right) \leq \sum_{v} \frac{p_{v, i}}{e^{t_{v} \delta_{i}}} \frac{\left(1-\rho_{v}\right) t_{v}}{t_{v}-\Lambda_{v}\left(M_{v}\left(t_{v}\right)-1\right)}\left(\frac{\alpha_{v, i} e^{\beta_{v, i} t_{v}}}{\alpha_{v, i}-t_{v}}\right)
$$


for any $0<t_{v}<\alpha_{v}, i, \rho_{v}=\Lambda_{v} \mathbb{E}\left[S_{v}\right]$, satisfying $M_{v}\left(t_{v}\right)<\infty$ and $t_{v}>\Lambda_{v}\left(M_{v}\left(t_{v}\right)-1\right)$.

Proof. Let $\mathbf{L}_{v, i}$ be the random sojourn time that app $i$ needs in order to send its update data to the BS if link $v$ is selected. From (5), we have $\Lambda_{v}=\sum_{i} \lambda_{i} p_{v, i}$. Then, using PollaczekKhinchine (PK) formula, $\mathbf{L}_{v, i}$ is given by

$$
\mathbb{E}\left[e^{t_{v} \mathbf{L}_{v, i}}\right]=\frac{\left(1-\rho_{v}\right) t_{v}}{t_{v}-\Lambda_{v}\left(M_{v}\left(t_{v}\right)-1\right)}\left(\frac{\alpha_{v, i} e^{\beta_{v, i} t_{v}}}{\alpha_{v, i}-t_{v}}\right),
$$

where $\rho_{v}=\Lambda_{v} \mathbb{E}\left[S_{v}\right]$ is the update intensity at link $v$. Using

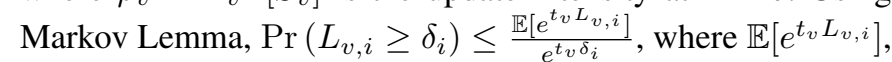
is given by the PK formula shown in (12), Theorem holds.

For the queuing system to be stable, we should have

$$
\Lambda_{v} \mathbb{E}\left[S_{v}\right]=\rho_{v} \leq 1, \forall v
$$

\section{AoI Characterization}

The results in [27] are for a single server and limited only to $\mathrm{M} / \mathrm{M} / 1$ system with identical updates. In this section, we generalize these results and consider general (i.e., shifted exponential) service time and allowing the updates to have different sizes.

Theorem 3. The expected AoI for an update from app i, i.e., $\mathbb{E}\left[\boldsymbol{A}_{i}\right]$, is thus given as

$$
\begin{aligned}
\mathbb{E}\left[\mathbf{A}_{i}\right] & =\sum_{v=1}^{V} \frac{p_{v, i}}{\nu_{v}\left(1-\rho_{v,-i}\right)} \times \\
& {\left[1+\frac{1}{\widetilde{\rho}_{v, i}}+\frac{\widetilde{\rho}_{v, i}^{2}}{1-\widetilde{\rho}_{v, i}}+\rho_{v,-i} \widetilde{\rho}_{v, i}^{2}\right] }
\end{aligned}
$$

where $\rho_{v, i}=p_{v, i} \lambda_{i} / \alpha_{v, i}, \quad \rho_{v,-i}=\rho_{v}-\rho_{v, i}, \quad \tilde{\rho}_{v, i}=$ $\rho_{v, i} /\left(1-\rho_{v,-i}\right), \rho_{v}=\Lambda_{v} / \nu_{v}$, given in (10) and satisfying $\rho_{v}<1$.

Proof. The update process for source $i$ is shown in Figure 3. In order to characterize the AoI for an update from app $i$, we first find the AoI assuming a given link $v, \mathbb{E}\left[\mathbf{A}_{v, i}\right]$. The conditioning and optimization over the decision variables $p_{v, i}$ will be considered later. Let $X_{v, i}$ be the inter-arrival time between app $i$ updates at link $v$ and $T_{v, i}$ is the total system time of delivered updates of app $i$ at link $v$. Further, let $T_{v, i}=$ $W_{v, i}+S_{v, i}$, where $W_{v, i}$ and $S_{v, i}$ be the respective waiting and service times for app $i$ at link $v$. From [27], the average AoI of an update for app $i$ at link $v$, in a stationary ergodic status updating system is given as

$$
\mathbb{E}\left[\mathbf{A}_{v, i}\right]=p_{v, i} \lambda_{i} \mathbb{E}\left[X_{v, i} W_{v, i}\right]+\frac{1}{\alpha_{v, i}}+\frac{1}{p_{v, i} \lambda_{i}}
$$

We note that $X_{v, i}$ and $W_{v, i}$ are negatively correlated since larger inter-arrival time can lead the queue to be empty, leading to a small $W_{v, i}$. To evaluate, $\mathbb{E}\left[X_{v, i} W_{v, i}\right]$, we need to take into account the flow of update packets $(j=1,2, \ldots)$ that belong to the same app $i$ under two possible scenarios. First, the inter-arrival time of the $j^{\text {th }}$ update of app $i$ at link $v$ is less than the system time of the preceding packet of app $i$
$\left(X_{v, i, j}<T_{v, i, j}\right)$. Note that $X_{v, i, j}$ is the inter-arrival time of an update $j$ belong to app $i$ at link $v$, while $T_{v, i, j}$ represents the total system time for an update $j$ of app $i$ at server $j$. Second, the case when $\left(X_{v, i, j}>T_{v, i, j}\right)$ which is the complementary scenario when $X_{v, i, j}$ is longer than the system time $T_{v, i, j}$. Under this partitioning, we have

$$
\begin{aligned}
& \mathbb{E}\left[X_{v, i, j} W_{v, i, j}\right]= \\
& \mathbb{E}\left[X_{v, i, j} W_{v, i, j} \mid X_{v, i, j}<T_{v, i, j}\right] \mathbb{P}\left(X_{v, i, j}<T_{v, i, j}\right)+ \\
& \mathbb{E}\left[X_{v, i, j} W_{v, i, j} \mid X_{v, i, j}>T_{v, i, j}\right] \mathbb{P}\left(X_{v, i, j}>T_{v, i, j}\right)
\end{aligned}
$$

The waiting time $W_{v, i, j}$ depends on two components: the residual time $\left(T_{v, i, j}-X_{v, i, j}\right)^{+}$and also on the workloads of updates from all other sources that arrive during the interarrival time of the $j^{\text {th }}$ arrival of app $i$. At steady state, the system times $T_{v, i, j}$ 's are stochastically equal in distributions, that is $T_{v, i} \stackrel{\stackrel{\mathrm{d}}{=}}{=} T_{v, i, j} \stackrel{\stackrel{\mathrm{d}}{=}}{=} T_{v, i, j-1} \stackrel{\mathrm{d}}{=} T_{v}$, where $\stackrel{\mathrm{d}}{=}$ denotes equal in distribution. Note that $\mathbb{P}\left(X_{v, i, j}<T_{v, i, j}\right)=\frac{p_{v, i} \lambda_{i}}{p_{v, i} \lambda_{i}+\nu_{v}-\Lambda_{v}}$, where $\nu_{v}=1 / \mathbb{E}\left[S_{v}\right]$ is the service rate at link $v$. Thus, we can show that [27]

$$
\begin{array}{r}
\mathbb{E}\left[X_{v, i, j} W_{v, i, j} \mid X_{v, i, j}<T_{v, i, j}\right]=\frac{1}{\nu_{v}^{2}} \times \\
{\left[\frac{2 \rho_{v,-i}}{\left(1-\rho_{v,-i}\right)^{2}}+\frac{1}{\left(1-\rho_{v,-i}\right)\left(1-\rho_{v}\right)}\right]}
\end{array}
$$

Similarly, we have

$$
\begin{aligned}
& \mathbb{E}\left[X_{v, i, j} W_{v, i, j} \mid X_{v, i, j}>T_{v, i, j}\right]= \\
& \left(\frac{1}{\nu_{v}-\Lambda_{v,-i}}+\frac{1}{p_{v, i} \lambda_{i}}\right)\left(\frac{\rho_{v,-i}}{\nu_{v}\left(1-\rho_{v,-i}\right)}\right)
\end{aligned}
$$

By Plugging (17) and (18) in (16) and (15), performing some averaging and algebraic manipulations, the theorem holds.

\section{JOINT OPTIMIZATION FOR AOI AND LTP}

\section{A. Optimization problem for AoI and LTP Trade-off}

In this subsection, we formulate an optimization problem to jointly optimize both metrics of AoI and LTP. Let $\boldsymbol{p}=$ $\left(p_{v, i}, \forall i \in\{1, \cdots, N\}\right.$, and $\left.v \in\{\mathrm{LTE}, \mathrm{WiFi}\}\right), \boldsymbol{q}=\left(q_{i}, \forall i\right)$ and $\boldsymbol{t}=\left(t_{v}\right)$. Let $\lambda=\sum_{i} \lambda_{i}$ and $\theta$ be the trade-off factor that determines the relative significance of tail latency and AoI in the optimization problem, where $\theta \in[0,1]$. Further, let $\omega_{i}$ reflects the weight (importance) of an app $i$. Without loss of generality, we define $\omega_{i}=\lambda_{i} / \lambda$, so larger arrival rates are weighted higher. This weighting gives our PSS policy more flexibility in scheduling app updates, where apps with higher weights (e.g., more time sensitive) can be prioritized over other apps that can tolerate more delay. Then, optimizing a convex combination of the two metrics (using trade-off factor $\theta$ ) can be formulated as follows.

$$
\begin{aligned}
\mathbf{m i n}_{\boldsymbol{t}, \boldsymbol{p}, \boldsymbol{q}} & \sum_{i=1}^{N}\left[(1-\theta) \frac{\lambda_{i}}{\lambda} \mathbb{E}\left[\boldsymbol{A}_{i}\right]+\theta \frac{\lambda_{i}}{\lambda} \operatorname{Pr}\left(\mathbf{L}_{i} \geq \delta\right)\right] \\
\text { s.t. } & (4),(5),(9),(11),(13),(14), \\
& \Lambda_{v}\left(M_{v}\left(t_{v}\right)-1\right)<t_{v}, \forall v
\end{aligned}
$$




$$
\begin{aligned}
& p_{v, i} \geq 0,0 \leq q_{i} \leq 1 \forall i, v \\
& 0<t_{v}<\alpha_{v, i}, \forall i, v
\end{aligned}
$$

Here, tuning $\theta=1$ to $\theta=0$, the solution for (19) spans the solutions that minimize the LTP to the ones that minimize the AoI of app updates. Next, we present our proposed algorithm.

\section{B. Proposed Online and Offline Algorithms}

The joint age and tail latency optimization problem given in (19)-(22) is optimized over three sets of variables: sampling probability $\boldsymbol{q}$, scheduling probabilities $\boldsymbol{p}$ and auxiliary parameters $\boldsymbol{t}$. Since the problem is non-convex, we propose an iterative algorithm to solve the problem. The proposed algorithm divides the problem into three subproblems (easy to handle) that optimize one variable while fixing the others. The three sub-problems are labeled as (i) Scheduling Optimization which optimizes $\boldsymbol{p}$ for a given $\boldsymbol{q}, \boldsymbol{t}$, (ii) Sampling Optimization which optimizes $\boldsymbol{q}$ for a given $\boldsymbol{p}, \boldsymbol{t}$, (iii) Auxiliary Variables Optimization which optimizes $\boldsymbol{t}$ for a given $\boldsymbol{q}, \boldsymbol{p}$. In order to solve the optimization problem, we use successive convex approximation to solve each sub-problem.

While our proposed PSS scheduling policy is optimized for an offline scenario, an online algorithm can be derived according to the stationary PSS decision probabilities. The arrival rates $\lambda_{i}$ can be estimated based on a window based method. In this setting, a window size of $\Delta W$ is chosen. Using these estimated arrival rates, the solution for the optimization problem in (19) gives the optimal offline PSS scheduling probabilities. According to these stationary scheduling probabilities, a randomized online policy can be obtained.

\section{Evaluation Results}

In this section, we evaluate our proposed algorithm for jointly optimizing the two metrics of AoI and LTP. We consider one mobile equipped with two links/interfaces, LTE and $\mathrm{WiFi}$, where each link has a different bandwidth (or speed). In particular, we set $\alpha_{v}=\{60,36\}$ so WiFi has $66 \%$ higher bandwidth than that of the LTE. Also, we set the shift parameters to $\beta_{v}=\{1.25,0.75\}$. Further, the update sizes $D_{i}$ of the different apps are assumed to follow a heavytailed Pareto distribution as it is a commonly used distribution for packet sizes [28], with shape factor of 2 and scale of 300 , respectively. Unless otherwise stated, we set $N=50$, $\delta_{i}=50, \omega_{i}=\lambda_{i} / \lambda, \Delta W=500$ and $\theta=0.01$. The update arrival rate of app $i$ is set to be $\tilde{\lambda}_{i}=\lambda_{b} /(i+1)$, where $\lambda_{b}$ is the base arrival rate, In order to initialize our algorithm, we assume uniform scheduling, $p_{v, i}=1 / V, t_{v}=0.01 \forall v$ and $q_{i} \forall i$ is chosen uniformly at random between 0 and 1 . The system performance of the developed joint AoI and LTP optimization is evaluated in both modes: offline and online. For the offline mode, we consider two baseline systems to compare with: Random Assignment (RA) and Proportionalservice-rate Assignments (PA) Policies. In the RA strategy, the sampling and scheduling probabilities $q_{i}, p_{i, v}$ 's are randomly chosen $\forall v, i$. Nonetheless, the choice of auxiliary variables $\mathbf{t}$ are optimized as explained in Section IV-B. In the PA strategy,

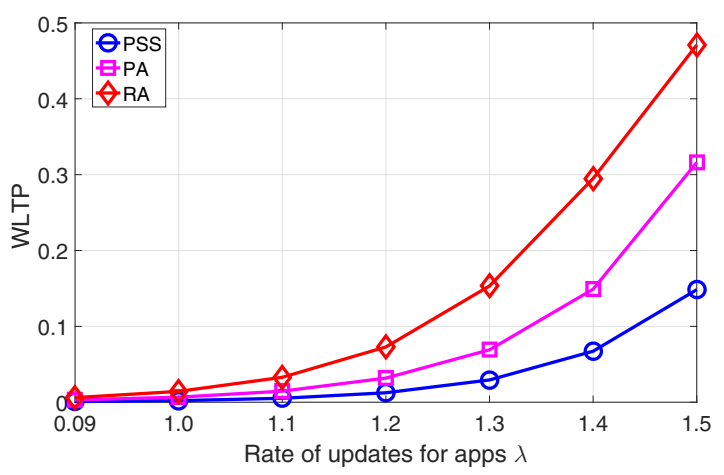

Fig. 4. WLTP versus $\lambda_{b}$.

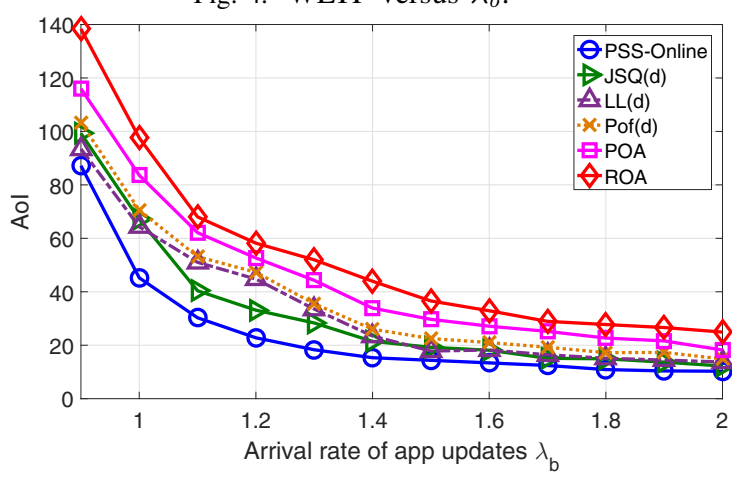

Fig. 5. AoI versus $\lambda_{b}$.

the scheduling decisions for app updates are chosen to be proportional to the service rates of the LTE and WiFi links. Also, the choice of auxiliary variables $(t, \forall v)$ and sampling rate $\boldsymbol{q}$ is optimized as explained in Section IV-B. In the online mode, we compare our algorithm with five baselines as described below: JSQ [22], LL(d) [23], Pof(d) [24], Random Online Assignment (ROA), and Proportional-service-rate Online Assignments (POA) Policies. For this mode, we set $V=10, d=4, \alpha_{v}=\alpha_{b} \times v / 2, \beta_{v}=\beta_{b} / v$, where $v=1, \ldots, 12, \alpha_{b}=60$ and $\beta_{b}=1.25$.

Fig. 4 shows an increasing trend in the value of the weighted LTP (WLTP) when the arrival rate increases. That is natural behavior since the system workload is increased. Nonetheless, PSS still performs the best (as compared to PA and RA) and achieves low LTP probabilities even with higher arrival rates.

As shown earlier (Fig. 1), there is a trade off between the mean AoI and the WLTP of the updates. In order to investigate such tradeoff, Table I shows the average AoI versus the WLTP for different values of $\theta$ ranging from $\theta=1$ to $\theta=0$. This table implies that a compromise between the two metrics can be obtained by our PSS algorithm by setting $\theta$ to an appropriate value. As expected, decreasing $\theta$ will decrease the AoI as there is more priority to minimize the AoI. More importantly, this table would serve as a look-up for the service provider to decide on an efficient trade-off point between the two metrics based on a desired performance level.

In Fig. 5, we study the performance of our online PSS algorithm and compare it with the state-of-art of queue-based scheduling algorithms as well as two competitive baselines. Different from our previous experiments and to better assess 
TABLE I

AGE AND WLTP TRADEOFF

\begin{tabular}{|c|c|c|}
\hline Tradeoff factor $\theta$ & Weighted AoI & Weighted LTP \\
\hline \hline$\theta=0.0$ & 0.1846 & 0.04180 \\
\hline$\theta=0.1$ & 0.1880 & 0.04175 \\
\hline$\theta=0.2$ & 0.1960 & 0.04155 \\
\hline$\theta=0.3$ & 0.3100 & 0.04105 \\
\hline$\theta=0.4$ & 0.3812 & 0.03915 \\
\hline$\theta=0.5$ & 0.5821 & 0.03261 \\
\hline$\theta=0.6$ & 0.6425 & 0.02719 \\
\hline$\theta=0.7$ & 0.8237 & 0.02342 \\
\hline$\theta=0.8$ & 1.1820 & 0.02121 \\
\hline$\theta=0.9$ & 1.3668 & 0.02081 \\
\hline$\theta=1.0$ & 1.6275 & 0.01915 \\
\hline
\end{tabular}

our online PSS policy, we consider the number of parallel links $V=12$ and then we select randomly $d=4$ links so that the update packet will join the lowest loaded link among the four. We first note that, besides its low-complexity, PSS still achieves the lowest age by efficiently exploiting all design control parameters including the scheduling and sampling probabilities. Further, queue-based policies, JSQ, Pof(d) and LL(d) do not consider the links speed when taking the decisions and also do not differentiate between app updates and thus not intelligently incorporate the update weight when taking the dispatching/sampling decisions. While our PSS algorithm optimizes the system parameters offline, this figure shows that an online version of our algorithm can be developed to keep track of the system dynamics and thus achieves an improved performance.

\section{CONCLUSIONS}

In this paper, we have looked at the problem of sampling and scheduling the information updates of mobile applications, while quantifying two quality of service measures: AoI and LTP. A novel scheduling policy is proposed. Further, we have derived closed form expressions for the LTP and AoI in M/G/1 systems, where shifted exponential service time is considered. Moreover, we have formulated an optimization problem to optimize a convex combination of weighted AoI and LTP. Our evaluation results outperforms the state of the art algorithms.

\section{REFERENCES}

[1] A. M. Bedewy, Y. Sun, and N. B. Shroff, "Optimizing data freshness, throughput, and delay in multi-server information-update systems," in Information Theory (ISIT), 2016 IEEE International Symposium on. IEEE, 2016, pp. 2569-2573.

[2] S. Kaul, M. Gruteser, V. Rai, and J. Kenney, "Minimizing age of information in vehicular networks," in Sensor, Mesh and Ad Hoc Communications and Networks (SECON), 2011 8th Annual IEEE Communications Society Conference on. IEEE, 2011, pp. 350-358.

[3] Y. Sun, E. Uysal-Biyikoglu, R. D. Yates, C. E. Koksal, and N. B. Shroff, "Update or wait: How to keep your data fresh," IEEE Transactions on Information Theory, vol. 63, no. 11, pp. 7492-7508, 2017.

[4] M. Costa, S. Valentin, and A. Ephremides, "On the age of channel state information for non-reciprocal wireless links," in Information Theory (ISIT), 2015 IEEE International Symposium on. IEEE, 2015, pp. 23562360.

[5] C. Kam, S. Kompella, G. D. Nguyen, J. E. Wieselthier, and A. Ephremides, "Information freshness and popularity in mobile caching," in Information Theory (ISIT), 2017 IEEE International Symposium on. IEEE, 2017, pp. 136-140.
[6] N. Lu, B. Ji, and B. Li, "Age-based scheduling: Improving data freshness for wireless real-time traffic," in Proceedings of the Eighteenth ACM International Symposium on Mobile Ad Hoc Networking and Computing. ACM, 2018, pp. 191-200.

[7] B. Zhou and W. Saad, "Joint status sampling and updating for minimizing age of information in the internet of things," arXiv preprint arXiv:1807.04356, 2018

[8] A. Alabbasi and V. Aggarwal, "Joint information freshness and completion time optimization for vehicular networks," arXiv preprint arXiv:1811.12924, 2018.

[9] S. Kaul, R. Yates, and M. Gruteser, "Real-time status: How often should one update?" in INFOCOM, 2012 Proceedings IEEE. IEEE, 2012, pp. 2731-2735.

[10] S. K. Kaul, R. D. Yates, and M. Gruteser, "Status updates through queues," in Information Sciences and Systems (CISS), 2012 46th Annual Conference on. IEEE, 2012, pp. 1-6.

[11] A. Elgabli, H. Khan, M. Krouka, and M. Bennis, "Reinforcement learning based scheduling algorithm for optimizing age of information in ultra reliable low latency networks," arXiv preprint arXiv:1811.06776, 2018.

[12] Z. Liu and B. Ji, "Towards the tradeoff between service performance and information freshness," arXiv preprint arXiv:1901.00826, 2019.

[13] R. Talak and E. Modiano, "Age-delay tradeoffs in single server systems," arXiv preprint arXiv:1901.04167, 2019.

[14] A. O. Al-Abbasi and V. Aggarwal, "Mean latency optimization in erasure-coded distributed storage systems," in IEEE INFOCOM 2018IEEE Conference on Computer Communications Workshops (INFOCOM WKSHPS). IEEE, 2018, pp. 432-437.

[15] A. O. Al-Abbasi, V. Aggarwal, and T. Lan, "TTLoC: Taming Tail Latency for Erasure-coded Cloud Storage Systems," IEEE Transactions on Network and Service Management, 2019.

[16] K. Gardner, S. Zbarsky, M. Velednitsky, M. Harchol-Balter, and A. Scheller-Wolf, "Understanding response time in the redundancy-d system," ACM SIGMETRICS Performance Evaluation Review, vol. 44, no. 2, pp. 33-35, 2016.

[17] F. Baccelli, A. Makowski, and A. Shwartz, "The fork-join queue and related systems with synchronization constraints: stochastic ordering and computable bounds," Advances in Applied Probability, pp. 629-660, 1989.

[18] W. Huajin, L. Jianhui, S. Zhihong, and Z. Yuanchun, "Approximations and bounds for (n, k) fork-join queues: A linear transformation approach," CoRR, vol. abs/1703.08337, 2017.

[19] Y.-P. Hsu, E. Modiano, and L. Duan, "Age of information: Design and analysis of optimal scheduling algorithms," in Information Theory (ISIT), 2017 IEEE International Symposium on. IEEE, 2017, pp. 561565.

[20] J. Dean and L. A. Barroso, "The tail at scale," Communications of the $A C M$, vol. 56, no. 2, pp. 74-80, 2013.

[21] Y. Xu, Z. Musgrave, B. Noble, and M. Bailey, "Bobtail: Avoiding long tails in the cloud." in NSDI, vol. 13, 2013, pp. 329-342.

[22] N. D. Vvedenskaya, R. L. Dobrushin, and F. I. Karpelevich, "Queueing system with selection of the shortest of two queues: An asymptotic approach," Problemy Peredachi Informatsii, vol. 32, no. 1, pp. 20-34, 1996.

[23] T. Hellemans and B. Van Houdt, "On the power-of-d-choices with least loaded server selection," Proceedings of the ACM on Measurement and Analysis of Computing Systems, vol. 2, no. 2, p. 27, 2018.

[24] L. Ying, R. Srikant, and X. Kang, "The power of slightly more than one sample in randomized load balancing," in Computer Communications (INFOCOM), 2015 IEEE Conference on. IEEE, 2015, pp. 1131-1139.

[25] R. D. Yates, M. Tavan, Y. Hu, and D. Raychaudhuri, "Timely cloud gaming," in INFOCOM 2017-IEEE Conference on Computer Coтmunications, IEEE. IEEE, 2017, pp. 1-9.

[26] F. Xu, F. Liu, and H. Jin, "Heterogeneity and interference-aware virtual machine provisioning for predictable performance in the cloud," IEEE Transactions on Computers, vol. 65, no. 8, pp. 2470-2483, Aug. 2016. [Online]. Available: doi.ieeecomputersociety.org/10.1109/ TC. 2015.2481403

[27] R. D. Yates and S. K. Kaul, "The age of information: Real-time status updating by multiple sources," IEEE Transactions on Information Theory, 2018.

[28] B. C. Arnold, Pareto distribution. Wiley Online Library, 2015. 